\title{
Influence of jaw tracking in intensity-modulated and volumetric-modulated arc radiotherapy for head and neck cancers: a dosimetric study
}

\author{
Karthick Raj Mani, MSc ${ }^{1}$ Sagar Upadhayay, MSc², K. J. Maria Das, PhD ${ }^{3}$ \\ ${ }^{1}$ Research and Development Centre, Bharathiar University, Tamilnadu, India; \\ ${ }^{2}$ Radiation Oncology, Kathmandu Cancer Center, Bhaktapur, Nepal; \\ ${ }^{3}$ Department of Radiotherapy, Sanjay Gandhi Postgraduate Institute of Medical Sciences, Uttar Pradesh, India
}

Purpose: To Study the dosimetric advantage of the Jaw tracking technique in intensity-modulated radiotherapy (IMRT) and volumetric-modulated arc therapy (VMAT) for Head and Neck Cancers.

Materials and Methods: We retrospectively selected 10 previously treated head and neck cancer patients stage (T1/T2, N1, $\mathrm{MO})$ in this study. All the patients were planned for IMRT and VMAT with simultaneous integrated boost technique. IMRT and VMAT plans were performed with jaw tracking (JT) and with static jaw (SJ) technique by keeping the same constraints and priorities for a particular patient. Target conformity, dose to the critical structures and low dose volumes were recorded and analyzed for IMRT and VMAT plans with and without JT for all the patients.

Results: The conformity index average of all patients followed by standard deviation $\left(\bar{x} \pm \sigma_{\bar{x}}\right)$ of the JT-IMRT, SJ-IMRT, JT-VMAT, and SJ-VMAT were $1.72 \pm 0.56,1.67 \pm 0.57,1.83 \pm 0.65$, and $1.85 \pm 0.64$, and homogeneity index were $0.059 \pm 0.05,0.064 \pm 0.05$, $0.064 \pm 0.04$, and $0.064 \pm 0.05$. JT-IMRT shows significant mean reduction in right parotid and left parotid shows of $7.64 \%(p<0.001)$ and 7.45\% ( $p<0.001)$ compare to SJ-IMRT. JT-IMRT plans also shows considerable dose reduction to thyroid, inferior constrictors, spinal cord and brainstem compared to the SJ-IMRT plans.

Conclusion: Significant dose reductions were observed for critical structure in the JT-IMRT compared to SJ-IMRT technique. In JTVMAT plans dose reduction to the critical structure were not significant compared to the SJ-IMRT due to relatively lesser monitor units.

Keywords: Jaw tracking, IMRT, VMAT, Dosimetric study

\section{Introduction}

Intensity-modulated radiotherapy (IMRT) and volumetricmodulated arc therapy (VMAT) became a standard treatment approach in head and neck (HEtN) cancer radiotherapy due to the ability of dose escalation to the tumor with reducing/ limiting the doses to critical structures. In HEtN cancers IMRT and VMAT published clinical results shows better outcomes in tumour control and quality of life compare to the conventional radiotherapy. The results do confirm that IMRT does decrease

Received 29 December 2016, Revised 8 February 2017, Accepted 22 February 2017.

Correspondence: K. J. Maria Das, PhD, Department of Radiotherapy, Sanjay Gandhi Postgraduate Institute of Medical Sciences, Rai Bareilly Road, Lucknow-226014, Uttar Pradesh India. Tel: +91-522-2494458, Fax: +91-522-2668644, E-mail: kjmariadas@hotmail.com

(c) This is an Open Access article distributed under the terms of the Creative Commons Attribution Non-Commercial License (http://creativecommons.org/ licenses/by-nc/4.0/) which permits unrestricted non-commercial use, distribution, and reproduction in any medium, provided the original work is properly cited.

www.e-roj.org 
xerostomia compared with conventional radiotherapy [1]. Low dose volume regions are a major worry in modulated radiotherapy because of its risk of secondary malignancies incidence, especially in the adult and pediatric patients. The prevalence of second malignancies after radiotherapy for pediatric and young adult populations is well established as one of the significant long-term sequelae of radiation treatment [2]. In sliding window IMRT technique the multileaf collimators (MLC) deliver doses by moving from bank ' $B$ ' towards ' $A$ ' bank with variable speed and dose rate with fixed jaws. Jaw tracking techniques offers to reduce the MLC transmission during the IMRT and VMAT, during the jaw tracking treatment the secondary jaws $\left(Y_{1}, Y_{2}, X_{1} \& t X_{2}\right)$ will dynamically move as close to the MLC segments to further minimize the MLC transmission. Typically values for the MLC transmission for the Millennium 120 leaf MLC (Varian Medical Systems, Palo Alto, CA, USA) were between $1.6 \%$ to $2.5 \%$ for beam energy $6 \mathrm{MV}$ to $18 \mathrm{MV} \mathrm{X}$-ray photon beams, with the use of jaw tracking by combining the MLC and secondary jaws we can further reduce the leakage radiation to negligible amount. According to the study by LoSasso et al. [3], the MLC transmission increases with increasing jaw field size and beam energy. Cadman et al. [4] found that the transmission through the jaw and the MLC together is smaller than $0.1 \%$.

TrueBeam medical linear accelerator (Varian Medical Systems) is equipped with jaw tracking technique to reduce the MLC transmission by dynamic tracking of the secondary jaws during the IMRT and VMAT delivery was used in this study. The jaws can move at the maximum speed of $2 \mathrm{~cm} /$ $\mathrm{s}$, during the IMRT or VMAT dose delivery as close as possible to the MLC aperture, and further minimizes leakage and transmission through the MLC leaves [5].

In this study we intent to estimate the dosimetric influence of the jaw tracking technique in HEtN cancers patients with sliding window IMRT and VMAT techniques to compare with standard static jaws sliding window IMRT and VMAT plans by evaluating the doses to the critical structures, low dose volumes by keeping the priorities and constraints same with comparable plan quality indexes.

\section{Materials and Methods}

We retrospectively selected ten previously treated H\&N cancer patients stage $(\mathrm{T} 1 / \mathrm{T} 2, \mathrm{~N} 1, \mathrm{M} 0)$ in this study. All the patients were planned for sliding window IMRT and VMAT with simultaneous integrated boost (SIB) technique to deliver a differential dose per fraction to the high, intermediate and low risk volume using a single plan. We intend to deliver 70 Gy to high risk volume, 64 Gy to intermediate risk volume and $56 \mathrm{~Gy}$ for the low risk volume in 35 fractions. All the IMRT/ VMAT plans were planned with flattened 6 MV photons using Millennium 120 leaf MLC with and without jaw tracking by keeping the same priorities for the target volumes and critical structures for a particular patient. Eclipse treatment planning system, ver. 11.0 (Varian Medical Systems), was used in this study. To standardize all the IMRT and VMAT plans with and without jaw tracking technique were normalized at the target mean of the high risk volume (planning target volume [PTV], $70 \mathrm{~Gy})$.

\section{Patient demographic data}

Ten patients with mean age of 66 years (range, 53 to 76 years) were retrospectively included in this study from our previously treated patient database. The selected H\&N cancer patients which mostly includes were tongue, larynx, pyriform fossa, hypopharynx, and tonsil. The demographic data of the patients were listed in the Table 1.

\section{CT simulation and delineation}

All the patients were immobilized with custom made 'S' type Thermoplastic IMRT Reinforced mask (CIVCO, Orange City, $\left.I_{A}, U S A\right)$ fixed with the 'S' type overlay board indexed to the couch. A computed tomography (CT) axial scans with a slice thickness of $2.5 \mathrm{~mm}$ were obtained for all the patients using GE Discovery 60016 slice PET/CT scanner (GE Healthcare, Waukesha, WI, USA). Once the patient CT data are acquired, the CT images were imported in DICOM format to Eclipse TPS ver. 11.0 (Varian Medical Systems). The body structure was segmented automatically by the treatment planning system. Different anatomical structures and regions of interests were delineated. Organs-at-risk (OARs; right parotid, left parotid,

Table 1. Patients' demographic data

\begin{tabular}{|c|c|c|c|}
\hline Patient no. & Age $(y r) / s e x$ & Diagnosis & Stage \\
\hline 1 & $56 / \mathrm{M}$ & Ca_Tongue & $\mathrm{T} 2, \mathrm{~N} 1, \mathrm{MO}$ \\
\hline 2 & $62 / F$ & Ca_Larynx & $\mathrm{T} 1, \mathrm{~N} 1, \mathrm{M} 0$ \\
\hline 3 & $63 / F$ & Ca_Larynx & $\mathrm{T} 2, \mathrm{NO}, \mathrm{MO}$ \\
\hline 4 & $70 / \mathrm{M}$ & Ca_Pyriform fossa & $\mathrm{T} 2, \mathrm{~N} 1, \mathrm{MO}$ \\
\hline 5 & $53 / \mathrm{M}$ & Ca_Tonsil & $\mathrm{T} 1, \mathrm{~N} 1, \mathrm{M} 0$ \\
\hline 6 & $59 / \mathrm{M}$ & Ca_Hypopharynx & $\mathrm{T} 2, \mathrm{~N} 1, \mathrm{M} 0$ \\
\hline 7 & $73 / F$ & Ca_Pyriform fossa & $\mathrm{T} 2, \mathrm{~N} 1, \mathrm{MO}$ \\
\hline 8 & $73 / \mathrm{M}$ & Ca_Tongue & $\mathrm{T} 2, \mathrm{~N} 1, \mathrm{MO}$ \\
\hline 9 & $76 / \mathrm{M}$ & Ca_Pyriform fossa & $\mathrm{T} 2, \mathrm{~N} 1, \mathrm{MO}$ \\
\hline 10 & $71 / F$ & Ca_Pyriform fossa & $\mathrm{T} 1, \mathrm{~N} 1, \mathrm{M} 0$ \\
\hline
\end{tabular}



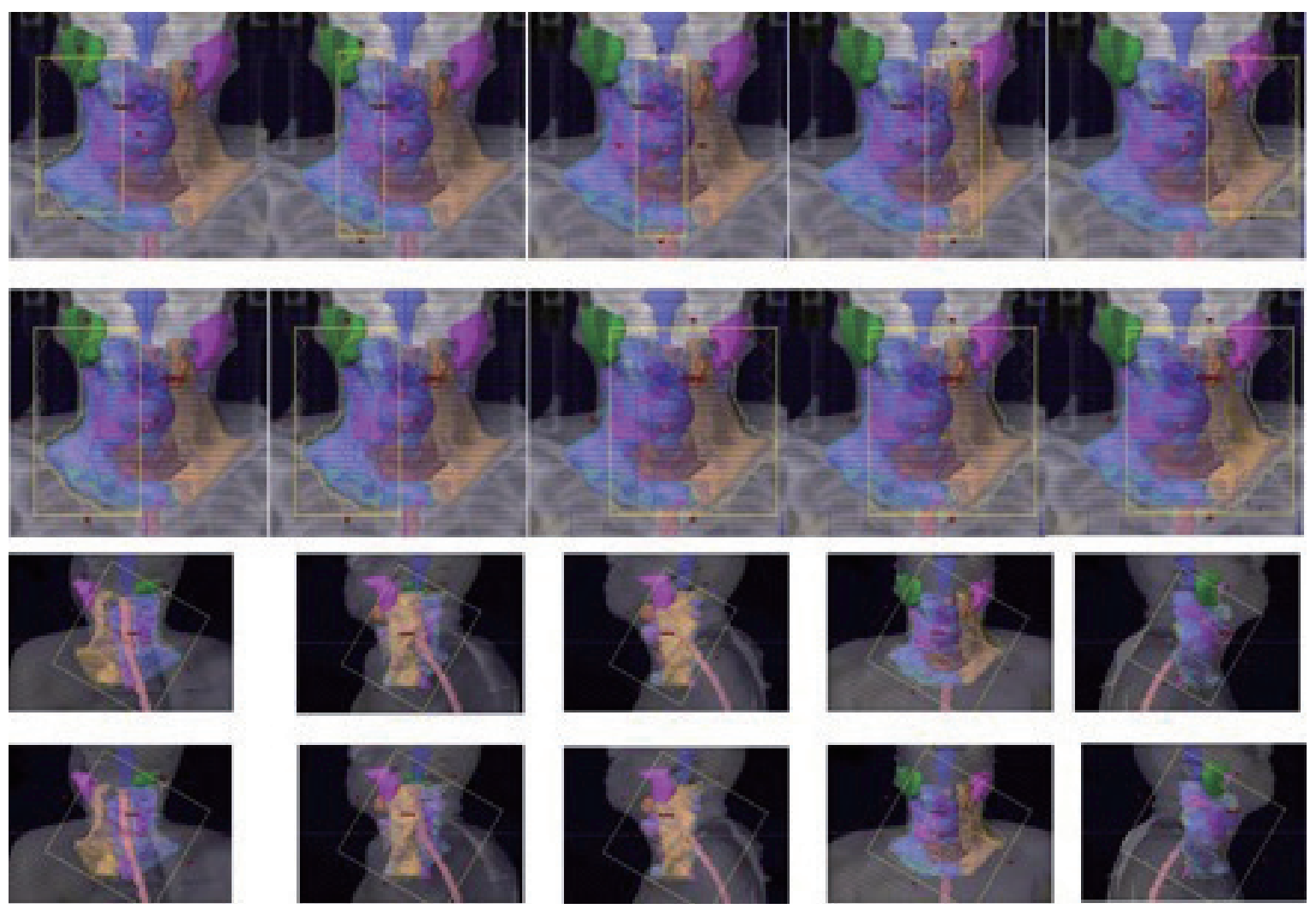

C

Fig. 1. (A) Gantry $0^{\circ}$ JT-IMRT field segments. (B) Gantry $0^{\circ} \mathrm{SJ}-$ IMRT field segments. (C) JT-VMAT arc segments. (D) SJ-VMAT arc segments. JT, jaw tracking; SJ, static jaw; IMRT, intensity-modulated radiotherapy; VMAT volumetric modulated arc therapy.

spinal cord, brainstem, thyroid, and inferior constrictor) were delineated by the physician slice by slice on the CT images for all the patients. PET/CT and magnetic resonance images (if available) were fused with the planning CT to define the gross tumor volume (GTV). An isotropic margin of $1 \mathrm{~cm}$ to $1.5 \mathrm{~cm}$ expansion from GTV were used to define the high risk CTV and manually edited from the anatomical boundaries (i.e., bone and air cavity) additional $0.4 \mathrm{~cm} 3 \mathrm{D}$ margin was given to generate the high risk PTV (HR-PTV) to account for the setup errors. Intermediate subclinical target volume and low risk subclinical target volume were also delineated and a setup margin was given to form the intermediate risk PTV (IR-PTV) and low risk PTV (LR-PTV).

\section{Treatment planning and optimization}

IMRT and VMAT plans for all the patients were done using SIB technique, which is to deliver a differential dose per fraction to the HR-PTV, IR-PTV, and LR-PTV using a single plan. We intend to deliver 70 Gy (2 Gy per fraction) to HR-PTV, 63 Gy (1.8 Gy per fraction) to IR-PTV and 56 Gy (1.6 Gy per fraction) to the LR-PTV in total 35 fractions.
The 6-MV photon flatten beam along with Millennium 120 leaf were used for the IMRT and VMAT planning optimization. IMRT and VMAT plans were performed with jaw tracking (JT) and with static jaw (SJ) technique by keeping the same constraints and priorities for the target volumes and critical structures for a particular patient. All the patients were planned with 7 fields SJ-IMRT by keeping the machine and optimization parameters identical. SJ-IMRT plans were duplicated to obtain JT-IMRT plans by enabling the JT technique and by recalculating the leaf motion calculator. VMAT plans with SJ and JT were planned with dual arc with $\pm 30^{\circ}$ collimator rotation with identical optimization parameters and dose constraints. The beams eye view (BEV) of SJ-IMRT, JT-IMRT, SJ-VMAT, and JT-VMAT are given below (Fig. 1).

All the IMRT plans were optimized using dose volume optimizer algorithm (DV0) ver.11.0 and the smart leaf motion calculator ver. 10.0 were used for converting the optimal fluence to actual fluence. The VMAT plans were optimized using the progressive resolution optimizer algorithm (PRO) ver.11.0. Dose calculation of both IMRT and VMAT plans with JT and SJ were calculated using analytical anisotropic algorithm 
(AAA) ver.11.0. All the SJ-IMRT, JT-IMRT, SJ-VMAT and JTVMAT plans were dose normalized to the HR-PTV mean for standardization of the plans. The dose constraints for the critical structures used in the optimization are listed in the Table 2.

\section{Planning evaluation}

ICRU 83 Report [6] released in 2010 used different concepts of plan evaluation parameters to evaluate the plans. To compare the IMRT and VMAT plans with SJ and JT technique, we used the ICRU 83 definition to determine the dose conformity and dose homogeneity. Dose conformity and homogeneity are independent specifications of the quality of the absorbed dose distribution. Dose conformity characterizes the degree to which the high dose region conforms to the target volume whereas dose homogeneity characterizes the uniformity of the absorbed dose within the target volume.

\section{1) Homogeneity index (HI):}

$$
H I=\frac{D_{20 \%}-D_{98 \%}}{D_{50 \%}}
$$

where, $D_{2 \%,} D_{98 \%,}$ and $D_{50 \%}$ are the volume received by $2 \%, 98 \%$ and $50 \%$, respectively. $\mathrm{HI}=0$ (zero) is ideal value.

Table 2. Dose constraints for the critical structures

\begin{tabular}{lr}
\hline \multicolumn{1}{c}{ Organ } & Dose volume constraints \\
\hline Brainstem & Max $<54 \mathrm{~Gy}$ \\
Right and left parotid & Mean $<26 \mathrm{~Gy}$ \\
Spinal cord & Max $<45 \mathrm{~Gy}$ \\
Thyroid & Mean $<50 \mathrm{~Gy}$ \\
Inferior constrictor & Mean $<50 \mathrm{~Gy}$ \\
\hline
\end{tabular}

2) Conformity index (CI): In 1993, Radiation Therapy Oncology Group recommended $\mathrm{Cl}$ as a ratio of the reference isodose volume to the target volume.

$$
C l_{\text {RTOG }}=\frac{V_{R I}}{T V}
$$

where, $V_{R I}$ reference isodose volume, and $T V$ is the target volume.

3) OAR: Mean and maximum doses were documented for the parallel and serial architecture structures, respectively, for both IMRT/NMAT plans with SJ and JT techniques. Additional dose parameters such as $V_{51}, V_{10}, V_{20}$ and $V_{30}$ (volume receiving at least $5 \mathrm{~Gy}, 10 \mathrm{~Gy}, 20 \mathrm{~Gy}$, and $30 \mathrm{~Gy}$, respectively) for both parotids, thyroid, spinal cord, brainstem and inferior constrictors were recorded. The dose changes to the whole body in the region of interest were also evaluated by comparing the $V_{51} V_{10}, V_{20}, V_{30}$ and mean dose to compare the low dose volume reduction in IMRT/VMAT plans with JT to the SJ technique. Dose conformity and dose heterogeneity were also calculated using the $\mathrm{HI}$ and $\mathrm{Cl}$ using the equations (1) and (2).

\section{Patient specific quality assurance}

Patient specific quality assurance for all the 10 patients with VMAT/IMRT plans with SJ and JT technique were verified using 2D array I'mRT MatriXX (Scanditronix Wellhofer, Freiburg, Germany) attached to the gantry head using the gantry mount. Verification plans were created using the Eclipse TPS and irradiated using the $2 \mathrm{D}$ ionization chamber array and compare the measured dose profiles with the Eclipse TPS using OmniPro IMRT software. All the 40 plans, each patient 4 plans (JT-IMRT, SJ-IMRT, JT-VMAT, and SJ-VMAT) were estimated

\begin{tabular}{|c|c|c|c|c|}
\hline Parameter & JT-IMRT & SJ-IMRT & JT-VMAT & SJ-VMAT \\
\hline $\mathrm{D}_{2 \%}(\mathrm{~Gy})$ & $71.951 \pm 0.04$ & $71.940 \pm 0.38$ & $72.580 \pm 1.11$ & $72.674 \pm 1.14$ \\
\hline$D_{50 \%}(G y)$ & $70.029 \pm 0.53$ & $70.030 \pm 0.57$ & $70.495 \pm 1.16$ & $70.500 \pm 1.18$ \\
\hline$D_{98 \%}(G y)$ & $67.793 \pm 0.54$ & $67.715 \pm 0.56$ & $68.055 \pm 1.46$ & $68.138 \pm 1.52$ \\
\hline $95 \%$ isodose volume $(\mathrm{mL})$ & $113.117 \pm 50.34$ & $110.185 \pm 51.48$ & $120.617 \pm 67.89$ & $122.003 \pm 68.97$ \\
\hline HR-PTV volume (mL) & \multicolumn{4}{|c|}{$65.711 \pm 41.49$} \\
\hline $\mathrm{Cl}_{\text {RTOG }}$ & $1.720 \pm 0.56$ & $1.670 \pm 0.57$ & $1.830 \pm 0.65$ & $1.850 \pm 0.64$ \\
\hline $\mathrm{HI}$ & $0.059 \pm 0.05$ & $0.064 \pm 0.05$ & $0.064 \pm 0.04$ & $0.064 \pm 0.05$ \\
\hline
\end{tabular}

Table 3. $\mathrm{Cl}$ and HI for HR-PTV

Values are presented as mean \pm standard deviation.

$\mathrm{Cl}$, conformity index; HI, homogeneity index; PTV, planning target volume; HR, high risk; JT, jaw tracking; SJ, static jaw; IMRT, intensity-modulated radiotherapy; VMAT volumetric-modulated arc therapy; RTOG, Radiation Therapy Oncology Group.

$D_{2 \%,} D_{98 \%}$ and $D_{50 \%}$ are the PTV volume received $2 \%, 98 \%$, and $50 \%$ dose, respectively, 
using the gamma evaluation method using 3\% dose difference and $3 \mathrm{~mm}$ distance to agreement (DTA) criteria.

\section{Statistical analysis}

The statistical data were presented as the average of all the patients followed by the standard deviation $\left(\bar{x} \pm \sigma_{\bar{x}}\right)$. Both the SJ and JT technique results were compared using the paired sample t-test was performed using the Microsoft Word/Excel version 2010 with $p<0.05$ considered as significant.

\section{Results}

\section{Radiation conformity index and dose homogeneity index}

The treatment plan quality has been compared using dose conformity and dose homogeneity parameters of two techniques are performed using $\mathrm{Cl}$ and $\mathrm{HI}$. The calculated $\mathrm{HI}$ and $\mathrm{Cl}$ of the HR-PTV for both two techniques IMRT and VMAT with and without JT are tabulated in Table 3. The average $\mathrm{Cl}(\overline{\mathrm{x}}$ $\left.\pm \sigma_{\bar{x}}\right)$ values were $1.72 \pm 0.56$ for JT-IMRT, $1.67 \pm 0.57$ for SJIMRT, $1.83 \pm 0.65$ for JT-VMAT, and $1.85 \pm 0.64$ for SJ-VMAT.

\section{Low dose volumes}

The comparison of the whole body means doses and low dose volume using JT-IMRT and SJ-IMRT are listed in Table 4. The JT-IMRT plans displayed significantly lower doses of $\mathrm{V}_{5}$ $V_{10}, V_{20}$, and $V_{30}(4.69 \%, 2.44 \%, 2.12 \%$, and $2.07 \%)$ volumes and reduced lower mean doses of the whole body by $2.34 \%$

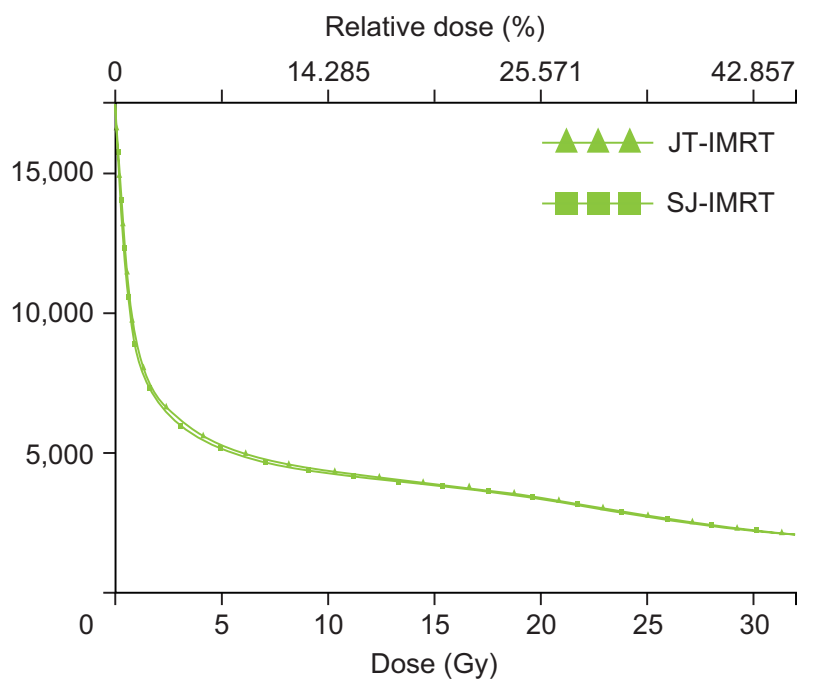

Fig. 2. Dose volume histogram comparison of low dose volume for JT-IMRT and SJ-IMRT. JT, jaw tracking; SJ, static jaw; IMRT, intensity-modulated radiotherapy. compared to the SJ-IMRT. The dose reduction in the $\mathrm{V}_{5}, \mathrm{~V}_{10}, \mathrm{~V}_{20}$ and $V_{30}$ and mean dose of the body were highly statistically significant $(p<0.001)$. The dose volume histogram comparison of low dose volumes for a patient between JT-IMRT and SJIMRT is shown in the Fig. 2.

The mean doses and low dose receiving volumes using JTVMAT and SJ-VMAT are listed in Table 5. The low dose volume $\left(\bar{x} \pm \sigma_{\bar{x}}\right)$ using JT-VMAT and SJ-VMAT were $9.101 \pm 2.53$ and $9.243 \pm 2.55$. The JT-VMAT plans displayed dose reduction in the $V_{51} V_{10}, V_{20}$, and $V_{30}(2.75 \%, 2.06 \%, 1.06 \%$, and $0.84 \%)$ volumes and lower mean doses of the whole body by $1.53 \%$ compared to the SJ-VMAT. We found a statistically significant dose reduction in the $V_{5}, V_{10}, V_{20}$, and mean dose of the body ( $p$ $<0.001$ ), but in $V_{30}$ volume was not statistically significant ( $p$ $=0.93$ ). The dose volume histogram comparison of low dose volumes for a patient between JT-VMAT and SJ-VMAT is shown in the Fig. 3.

\section{Right parotid}

Right parotid mean dose and the volumes of $V_{5}, V_{10}, V_{20}$, and $V_{30}$ for the JT-IMRT and SJ-IMRT were listed in Table 4. The JTIMRT plans shows a significant reduction in low dose receiving volume and mean dose. The mean dose reduction is $7.64 \%$ in JT-IMRT compare to the SJ-IMRT technique. We also found a statistical significant $(p<0.01)$ in the mean dose and $V_{5}, V_{10}$ and $\mathrm{V}_{30}$ volumes.

The mean doses to right parotid and volumes of $V_{5}, V_{10}$ $V_{20}$, and $V_{30}$ for the JT-VMAT and SJ-VMAT were listed in Table

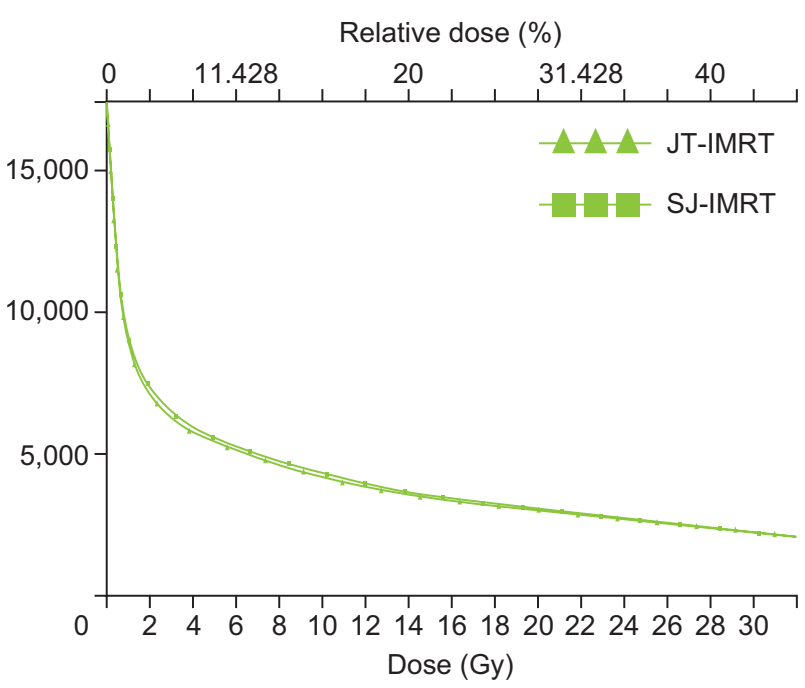

Fig. 3. Dose volume histogram comparison of low dose volume for JT-VMAT and SJ-VMAT. JT, jaw tracking; SJ, static jaw; VMAT, volumetric modulated arc radiotherapy. 
Table 4. Dose comparison and statistical significance of low dose volume and OARs between JT-IMRT and SJ-IMRT

\begin{tabular}{|c|c|c|c|c|}
\hline Organ & JT-IMRT & SJ-IMRT & Difference (\%) & $p$-value \\
\hline \multicolumn{5}{|l|}{ Body } \\
\hline$V_{5}(m L)$ & $4,295.57 \pm 702.49$ & $4,507.37 \pm 719.62$ & 4.69 & $<0.001$ \\
\hline$V_{10}(\mathrm{~mL})$ & $3,664.47 \pm 563.38$ & $3,756.01 \pm 547.35$ & 2.44 & $<0.001$ \\
\hline$V_{20}(\mathrm{~mL})$ & $2,964.47 \pm 431.43$ & $3,028.83 \pm 438.78$ & 2.12 & $<0.001$ \\
\hline $\mathrm{V}_{30}(\mathrm{~mL})$ & $2,196.93 \pm 311.07$ & $2,243.42 \pm 312.88$ & 2.07 & $<0.001$ \\
\hline Mean (Gy) & $9.16 \pm 2.57$ & $9.38 \pm 2.62$ & 2.34 & $<0.001$ \\
\hline \multicolumn{5}{|l|}{ Right parotid } \\
\hline$V_{5}(m L)$ & $17.80 \pm 5.96$ & $18.86 \pm 5.89$ & 5.71 & 0.009 \\
\hline$V_{10}(\mathrm{~mL})$ & $14.95 \pm 6.52$ & $15.79 \pm 6.67$ & 5.28 & $<0.001$ \\
\hline $\mathrm{V}_{20}(\mathrm{~mL})$ & $7.69 \pm 2.74$ & $8.03 \pm 3.12$ & 4.29 & 0.428 \\
\hline$V_{30}(\mathrm{~mL})$ & $5.56 \pm 2.39$ & $5.76 \pm 2.42$ & 3.57 & 0.016 \\
\hline Mean (Gy) & $19.96 \pm 3.95$ & $21.61 \pm 4.04$ & 7.64 & $<0.001$ \\
\hline \multicolumn{5}{|l|}{ Left parotid } \\
\hline$V_{5}(\mathrm{~mL})$ & $16.41 \pm 7.08$ & $18.84 \pm 7.04$ & 12.91 & 0.007 \\
\hline$V_{10}(\mathrm{~mL})$ & $14.81 \pm 6.43$ & $16.30 \pm 7.03$ & 9.15 & $<0.001$ \\
\hline$V_{20}(\mathrm{~mL})$ & $7.51 \pm 3.23$ & $8.17 \pm 3.59$ & 8.16 & 0.029 \\
\hline$V_{30}(\mathrm{~mL})$ & $5.80 \pm 2.90$ & $6.00 \pm 2.93$ & 3.33 & $<0.001$ \\
\hline Mean (Gy) & $20.11 \pm 2.72$ & $21.72 \pm 2.95$ & 7.45 & $<0.001$ \\
\hline \multicolumn{5}{|l|}{ Thyroid } \\
\hline$V_{5}(m L)$ & $14.86 \pm 6.89$ & $14.96 \pm 6.89$ & 0.70 & 0.360 \\
\hline $\mathrm{V}_{10}(\mathrm{~mL})$ & $14.86 \pm 6.89$ & $14.96 \pm 6.89$ & 0.70 & 0.200 \\
\hline $\mathrm{V}_{20}(\mathrm{~mL})$ & $14.80 \pm 6.90$ & $14.96 \pm 6.89$ & 1.06 & 0.120 \\
\hline $\mathrm{V}_{30}(\mathrm{~mL})$ & $12.06 \pm 5.34$ & $12.31 \pm 5.57$ & 2.03 & 0.240 \\
\hline Mean (Gy) & $44.61 \pm 8.45$ & $45.25 \pm 8.26$ & 1.40 & 0.003 \\
\hline \multicolumn{5}{|l|}{ Inferior constrictor } \\
\hline$V_{5}(\mathrm{~mL})$ & $7.27 \pm 2.97$ & $7.42 \pm 3.01$ & 2.07 & 0.090 \\
\hline$V_{10}(\mathrm{~mL})$ & $6.98 \pm 2.81$ & $7.12 \pm 2.84$ & 2.02 & 0.160 \\
\hline $\mathrm{V}_{20}(\mathrm{~mL})$ & $6.88 \pm 2.71$ & $6.91 \pm 2.73$ & 0.41 & 0.139 \\
\hline $\mathrm{V}_{30}(\mathrm{~mL})$ & $6.61 \pm 2.99$ & $6.66 \pm 2.76$ & 0.83 & 0.780 \\
\hline Mean (Gy) & $43.29 \pm 8.84$ & $44.32 \pm 8.69$ & 2.32 & $<0.001$ \\
\hline \multicolumn{5}{|l|}{ Spinal cord } \\
\hline$V_{5}(\mathrm{~mL})$ & $25.84 \pm 5.06$ & $27.64 \pm 5.07$ & 6.49 & 0.006 \\
\hline $\mathrm{V}_{10}(\mathrm{~mL})$ & $25.29 \pm 5.02$ & $26.76 \pm 5.19$ & 5.49 & 0.045 \\
\hline $\mathrm{V}_{20}(\mathrm{~mL})$ & $25.38 \pm 4.94$ & $26.54 \pm 4.95$ & 4.39 & 0.015 \\
\hline$V_{30}(\mathrm{~mL})$ & $22.57 \pm 7.01$ & $23.66 \pm 6.73$ & 4.61 & $<0.001$ \\
\hline Mean (Gy) & $30.68 \pm 3.99$ & $32.73 \pm 3.67$ & 6.25 & $<0.001$ \\
\hline Max (Gy) & $44.44 \pm 3.41$ & $46.76 \pm 3.49$ & 4.96 & 0.065 \\
\hline \multicolumn{5}{|l|}{ Brainstem } \\
\hline$V_{5}(m L)$ & $8.07 \pm 6.88$ & $8.83 \pm 7.21$ & 8.66 & 0.003 \\
\hline $\mathrm{V}_{10}(\mathrm{~mL})$ & $5.94 \pm 5.45$ & $6.42 \pm 5.92$ & 7.44 & 0.015 \\
\hline$V_{20}(\mathrm{~mL})$ & $3.86 \pm 3.35$ & $4.09 \pm 3.57$ & 5.72 & 0.005 \\
\hline $\mathrm{V}_{30}(\mathrm{~mL})$ & $2.73 \pm 2.13$ & $2.92 \pm 2.54$ & 6.43 & 0.068 \\
\hline Mean (Gy) & $7.90 \pm 4.22$ & $8.54 \pm 4.62$ & 7.49 & 0.002 \\
\hline $\operatorname{Max}(G y)$ & $41.99 \pm 9.32$ & $43.17 \pm 8.53$ & 2.72 & 0.003 \\
\hline
\end{tabular}

Values are presented as mean \pm standard deviation.

OAR, organ-at-risk; SJ, static jaw; JT, jaw tracking; IMRT, intensity-modulated radiotherapy. 
Table 5. Dose comparison and statistical significance of low dose volume and OARs between JT-VMAT and SJ-VMAT

\begin{tabular}{|c|c|c|c|c|}
\hline Organ & JT-IMRT & SJ-IMRT & Difference (\%) & $p$-value \\
\hline \multicolumn{5}{|l|}{ Body } \\
\hline $\mathrm{V}_{5}(\mathrm{~mL})$ & $4,539.00 \pm 720.00$ & $4,667.50 \pm 715.71$ & 2.75 & $<0.001$ \\
\hline $\mathrm{V}_{10}(\mathrm{~mL})$ & $3,599.63 \pm 556.21$ & $3,675.35 \pm 588.82$ & 2.06 & $<0.001$ \\
\hline $\mathrm{V}_{20}(\mathrm{~mL})$ & $2,768.38 \pm 379.59$ & $2,798.18 \pm 376.56$ & 1.06 & 0.009 \\
\hline $\mathrm{V}_{30}(\mathrm{~mL})$ & $2,235.93 \pm 307.69$ & $2,254.90 \pm 316.18$ & 0.84 & 0.930 \\
\hline Mean (Gy) & $9.10 \pm 2.53$ & $9.24 \pm 2.55$ & 1.53 & $<0.001$ \\
\hline \multicolumn{5}{|l|}{ Right parotid } \\
\hline $\mathrm{V}_{5}(\mathrm{~mL})$ & $18.64 \pm 5.85$ & $19.21 \pm 5.62$ & 2.93 & 0.240 \\
\hline$V_{10}(\mathrm{~mL})$ & $14.89 \pm 7.01$ & $15.34 \pm 6.88$ & 2.94 & 0.050 \\
\hline $\mathrm{V}_{20}(\mathrm{~mL})$ & $8.85 \pm 4.11$ & $8.81 \pm 3.94$ & 0.43 & 0.710 \\
\hline $\mathrm{V}_{30}(\mathrm{~mL})$ & $6.23 \pm 2.55$ & $6.27 \pm 2.58$ & 0.57 & 0.300 \\
\hline Mean (Gy) & $21.94 \pm 5.34$ & $22.08 \pm 5.10$ & 0.59 & 0.360 \\
\hline \multicolumn{5}{|l|}{ Left parotid } \\
\hline $\mathrm{V}_{5}(\mathrm{~mL})$ & $18.02 \pm 6.71$ & $18.78 \pm 6.83$ & 4.05 & 0.320 \\
\hline$V_{10}(\mathrm{~mL})$ & $13.49 \pm 6.09$ & $13.89 \pm 5.92$ & 2.90 & 0.030 \\
\hline $\mathrm{V}_{20}(\mathrm{~mL})$ & $7.15 \pm 3.11$ & $7.20 \pm 3.09$ & 0.79 & 0.490 \\
\hline $\mathrm{V}_{30}(\mathrm{~mL})$ & $4.67 \pm 2.59$ & $4.72 \pm 2.59$ & 0.93 & 0.630 \\
\hline Mean (Gy) & $19.37 \pm 4.89$ & $19.48 \pm 4.79$ & 0.55 & 0.260 \\
\hline \multicolumn{5}{|l|}{ Thyroid } \\
\hline $\mathrm{V}_{5}(\mathrm{~mL})$ & $14.96 \pm 6.89$ & $14.97 \pm 6.89$ & 0.05 & 0.320 \\
\hline $\mathrm{V}_{10}(\mathrm{~mL})$ & $14.96 \pm 6.89$ & $14.97 \pm 6.89$ & 0.05 & 0.320 \\
\hline $\mathrm{V}_{20}(\mathrm{~mL})$ & $14.44 \pm 6.61$ & $14.53 \pm 6.49$ & 0.58 & 0.170 \\
\hline $\mathrm{V}_{30}(\mathrm{~mL})$ & $11.88 \pm 5.45$ & $11.94 \pm 5.35$ & 0.51 & 0.230 \\
\hline Mean (Gy) & $44.23 \pm 7.84$ & $44.75 \pm 7.63$ & 1.16 & 0.030 \\
\hline \multicolumn{5}{|l|}{ Inferior constrictor } \\
\hline $\mathrm{V}_{5}(\mathrm{~mL})$ & $7.61 \pm 3.19$ & $7.65 \pm 3.25$ & 0.56 & 0.370 \\
\hline$V_{10}(\mathrm{~mL})$ & $7.33 \pm 2.83$ & $7.39 \pm 2.83$ & 0.79 & 0.240 \\
\hline $\mathrm{V}_{20}(\mathrm{~mL})$ & $6.84 \pm 2.75$ & $6.90 \pm 2.74$ & 0.78 & 0.370 \\
\hline $\mathrm{V}_{30}(\mathrm{~mL})$ & $6.52 \pm 3.02$ & $6.52 \pm 3.08$ & 0.05 & 0.980 \\
\hline Mean (Gy) & $43.25 \pm 8.92$ & $44.15 \pm 9.45$ & 2.03 & 0.450 \\
\hline \multicolumn{5}{|l|}{ Spinal cord } \\
\hline $\mathrm{V}_{5}(\mathrm{~mL})$ & $27.54 \pm 5.07$ & $27.84 \pm 5.09$ & 1.10 & 0.120 \\
\hline$V_{10}(\mathrm{~mL})$ & $27.11 \pm 5.04$ & $27.29 \pm 4.89$ & 0.63 & 0.580 \\
\hline $\mathrm{V}_{20}(\mathrm{~mL})$ & $26.23 \pm 4.84$ & $26.40 \pm 4.46$ & 0.63 & 0.490 \\
\hline $\mathrm{V}_{30}(\mathrm{~mL})$ & $24.81 \pm 4.60$ & $24.90 \pm 1.59$ & 0.37 & 0.012 \\
\hline Mean (Gy) & $33.39 \pm 4.29$ & $33.66 \pm 4.35$ & 0.81 & 0.640 \\
\hline Max (Gy) & $45.08 \pm 1.43$ & $45.16 \pm 1.60$ & 0.18 & 0.079 \\
\hline \multicolumn{5}{|l|}{ Brainstem } \\
\hline $\mathrm{V}_{5}(\mathrm{~mL})$ & $11.88 \pm 7.27$ & $12.10 \pm 7.96$ & 1.85 & 0.490 \\
\hline $\mathrm{V}_{10}(\mathrm{~mL})$ & $5.79 \pm 5.23$ & $5.88 \pm 5.69$ & 1.64 & 0.550 \\
\hline $\mathrm{V}_{20}(\mathrm{~mL})$ & $3.69 \pm 3.44$ & $3.73 \pm 3.58$ & 0.99 & 0.670 \\
\hline $\mathrm{V}_{30}(\mathrm{~mL})$ & $2.69 \pm 2.64$ & $2.72 \pm 2.83$ & 0.84 & 0.710 \\
\hline Mean (Gy) & $8.71 \pm 4.20$ & $9.04 \pm 4.68$ & 3.66 & 0.520 \\
\hline $\operatorname{Max}(G y)$ & $42.10 \pm 10.96$ & $42.68 \pm 3.25$ & 1.38 & 0.470 \\
\hline
\end{tabular}

Values are presented as mean \pm standard deviation.

OAR, organ-at-risk; SJ, static jaw; JT, jaw tracking; VMAT, volumetric modulated arc therapy. 


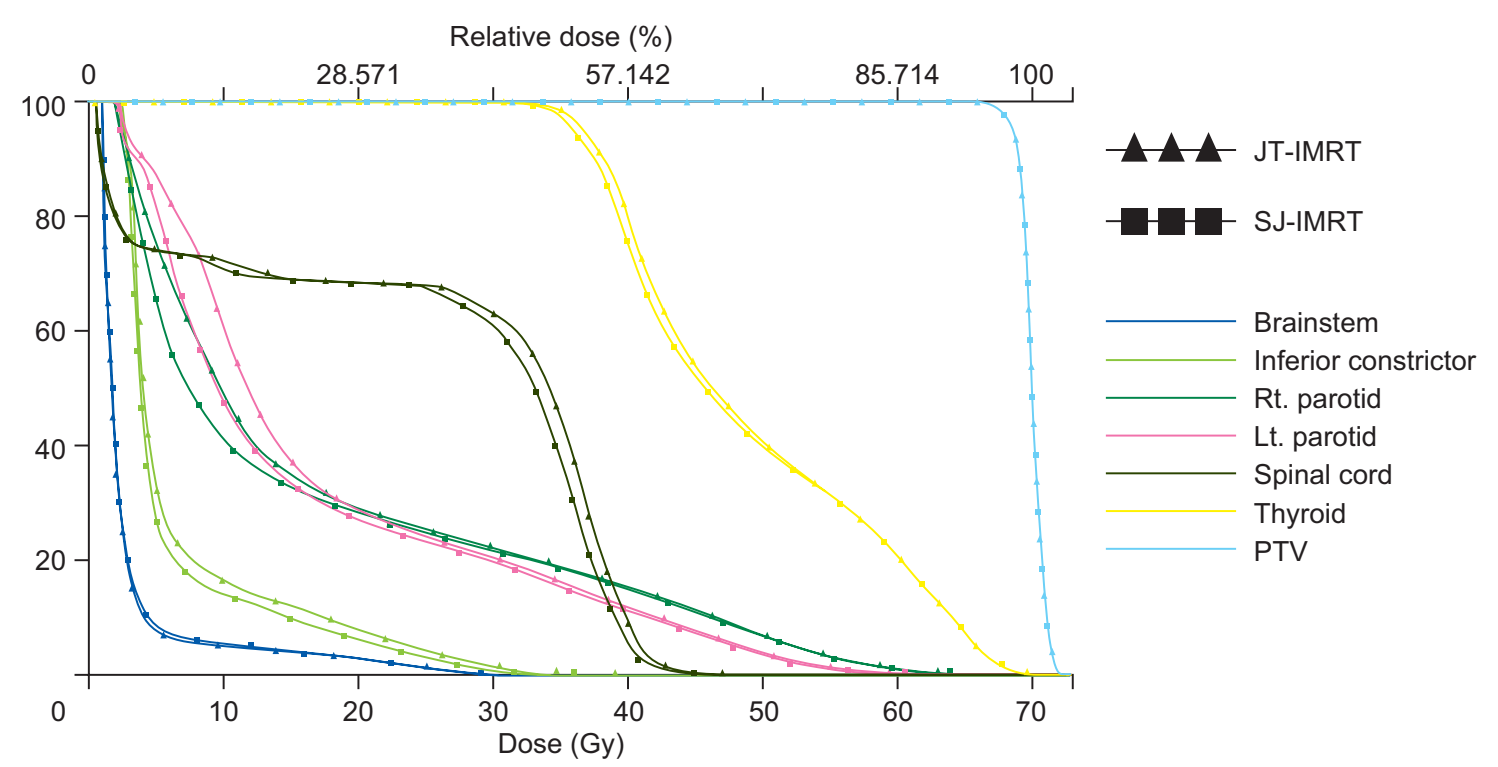

Fig. 4. Dose volume histogram comparison of OAR's for JT-IMRT and SJ-IMRT. OAR, organ-at-risk; JT, jaw tracking; SJ, static jaw; IMRT, intensity-modulated radiotherapy; PVT, planning target volume.

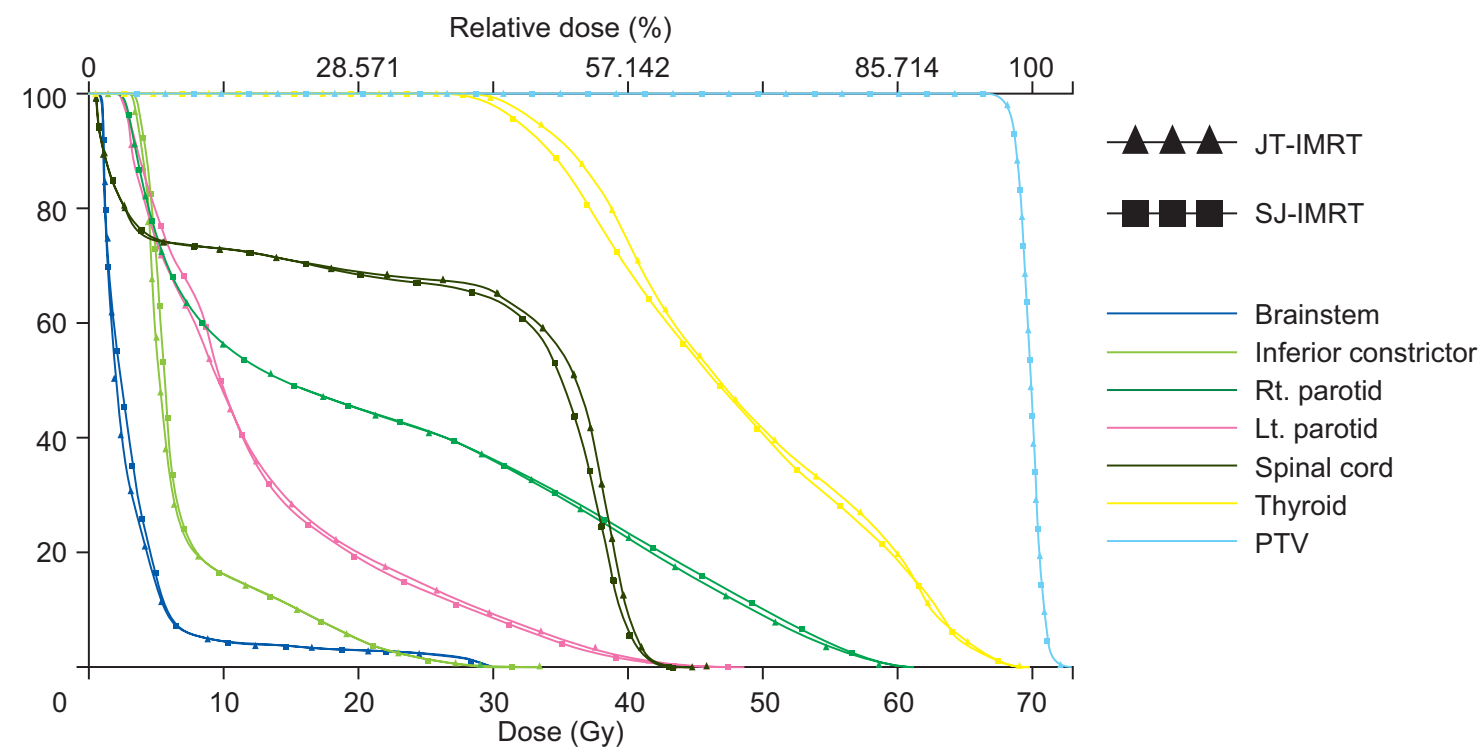

Fig. 5. Dose Volume Histogram comparison of OAR's for JT-VMAT and SJ-VMAT. OAR, organ-at-risk; JT, jaw tracking; SJ, static jaw; VMAT, volumetric modulated arc therapy; PVT, planning target volume.

5. The JT-VMAT plan displayed slight reduction in low dose receiving volume and mean dose $(p=0.36)$ and it is not statistically significant. The mean dose reduction is only $0.59 \%$ in JT-VMAT. The dose reduction is not appreciable in VMAT as compared to IMRT with JT, but $\mathrm{V}_{5}$ and $\mathrm{V}_{10}$ volumes shows around $2.9 \%$ reduction in JT-VMAT compared to SJ-VMAT.

\section{Left parotid}

The JT-IMRT plan displayed dose reduction in low dose receiving volume and mean dose compared to SJ-IMRT. The mean dose reduction is $7.45 \%(p<0.001)$ in JT-IMRT. The low dose volume $V_{5}$ and $V_{10}$ were significantly reduced by $12.91 \%$ and 9.15\%. The $V_{51} V_{10}$, and $V_{30}$ shows a statistically significant ( $p$ $=0.05$ ) for JT-IMRT compared to the SJ-IMRT.

The left parotid mean doses and the volume receiving doses of at least $5 \mathrm{~Gy}, 10 \mathrm{~Gy}, 20 \mathrm{~Gy}$ and $30 \mathrm{~Gy}$ for the JT-VMAT and SJ-VMAT are listed in Table 5. The JT-VMAT plan resulted in dose reduction in low dose receiving volumes and mean dose. 
The mean dose reduction is $0.55 \%(p=0.26)$ in JT-VMAT compared to SJ-VMAT. The dose reduction is not appreciable in VMAT as compared to IMRT with JT, but $\mathrm{V}_{5}$ and $\mathrm{V}_{10}$ were reduced to $4.05 \%$ and $2.9 \%$, respectively, in JT-VMAT compared to SJ-VMAT.

\section{Thyroid}

The statistical data of the thyroid for JT-IMRT and SJ-IMRT were listed in Table 4. The $V_{5}, V_{10}, V_{20}, V_{30}$, and mean dose of thyroid in JT-IMRT plans are lower than the corresponding values of the SJ-IMRT plans, the mean reduction were $0.70 \%$, $0.70 \%, 1.06 \%, 2.03 \%$, and $1.40 \%$ respectively. We found a statistically significant ( $p=0.003$ ) for the mean dose of JTIMRT compare to SJ-IMRT.

The $V_{5}, V_{10}, V_{20}, V_{30}$, and mean dose of thyroid in JT-VMAT plans are slightly lower than the corresponding values of the SJ-VMAT plans, the mean reduction were 0.047\%, 0.047\%, $0.58 \%, 0.51 \%$, and $1.16 \%$, respectively, and they are not of statistically significant.

\section{Inferior constrictor}

The statistical data of inferior constrictor for JT-IMRT and SJIMRT were listed in Table 4. The $\mathrm{V}_{5}, \mathrm{~V}_{10}, \mathrm{~V}_{20}, \mathrm{~V}_{30}$, and mean dose of inferior constrictor in JT-IMRT plans are lower than the corresponding values of the SJ-IMRT plans, the mean reduction was 2.07\%, 2.02\%, 0.41\%, 0.83\%, and 2.32\%, respectively. We found a statistically significant $(p<0.001)$ for the mean dose of JT-IMRT compared to SJ-IMRT.

The $V_{5}, V_{10}, V_{20}, V_{30}$, and mean dose of inferior constrictor in JT-VMAT plans were lower than the corresponding values of the SJ-VMAT plans, the mean reduction was 0.56\%, 0.79\%, $0.78 \%, 0.05 \%$, and $2.03 \%$, respectively (Table 5 ). There is no significant difference in low dose receiving volume and the mean dose volumes.

\section{Spinal cord}

The low dose volume, mean and maximum doses to spinal cord were listed for the JT-IMRT and SJ-IMRT in Table 4. The JTIMRT plans displayed reduction in low dose receiving volume, maximum and mean dose. The dose reduction for low dose volume were $V_{5}(6.49 \%), V_{10}(5.49 \%), V_{20}(4.39 \%), V_{30}(4.61 \%)$, mean dose (6.25\%), and maximum dose (4.96\%). The mean dose reduction were statistically significant $(p<0.001)$ for JTIMRT compared to SJ-IMRT.

The mean and maximum doses to spinal cord and the volume receiving doses of at least $5 \mathrm{~Gy}, 10 \mathrm{~Gy}, 20 \mathrm{~Gy}$, and 30 Gy for the JT-VMAT and SJ-VMAT were listed in Table 5. The JT-
VMAT plans displayed reduction in low dose receiving volume, maximum and mean dose. The dose reduction for low dose volume were $V_{5}(1.10 \%), V_{10}(0.63 \%), V_{20}(0.63 \%), V_{30}(0.37 \%)$, mean dose $(0.81 \%)$, and maximum dose $(0.18 \%)$.

\section{Brainstem}

Table 4 lists the mean and maximum doses to brainstem and also shows the volume receiving doses of at least $5 \mathrm{~Gy}$, $10 \mathrm{~Gy}, 20 \mathrm{~Gy}$, and $30 \mathrm{~Gy}$ for the JT-IMRT and SJ-IMRT plans. Significant reduction achieved with the JT-IMRT plan compared to SJ-IMRT plans. The JT-IMRT plans displayed reduction in low dose receiving volume, maximum and mean doses. The dose reduction for low dose volume were $V_{5}(8.66 \%), V_{10}$ (7.44\%), $V_{20}(5.72 \%), V_{30}(6.43 \%)$, mean dose (7.49\%), and maximum dose by (2.72\%). The mean and maximum has a significant dose reduction with $p=0.002$ and $p=0.003$, respectively, for JT-IMRT compared to SJ-IMRT.

JT-VMAT shows dose reduction compared to SJ-VMAT plans. The JT-VMAT plans displayed reduction in low dose receiving volume, maximum and mean dose. The dose reduction for low dose volume were $V_{5}(1.85 \%), V_{10}(1.64 \%), V_{20}(0.99 \%), V_{30}$ $(0.84 \%)$, mean dose $(3.66 \%)$, and maximum dose (1.38\%), but they are not of statistically significant.

Fig. 4 illustrates the dose volume histogram comparison of OAR's and HR-PTV for JT-IMRT and SJ-IMRT; it shows that JT-IMRT has a clear evidence of decreases in the low dose volume of the OAR especially $V_{5}$ and $V_{10}$. This study shows that the mean values of the maximum, mean doses and low dose receiving volume to the spinal cord, brainstem, both parotid, thyroid, inferior constrictor and body are significantly reduced maximum up to $12.91 \%$ in the JT plans. The reduction values are depending on the volume and location of the OARs. Fig. 5 shows the dose volume histogram comparison of OAR's and PTV for the JT-VMAT and SJ-VMAT, there was a slight reduction in the low dose volume region for the OAR's.

Patient specific quality assurance for all the JT and SJ IMRT/VMAT plans (40 plans) were performed by comparing the treatment planning system fluence and the measured fluence in the machine and evaluated using gamma evaluation method with $3 \mathrm{~mm}$ DTA and 3\% dose difference criteria. All the plans were passed with more than 97\% pixel within the gamma evaluation criteria. This shows the difference between dose calculation and measurements are within the tolerance.

\section{Discussion and Conclusion}

In the present study, IMRT and VMAT plans were compared 
with IMRT and VMAT with and without JT technique. Plan quality was assessed by comparing $\mathrm{HI}$ and $\mathrm{Cl}$. The target coverage for HR-PTV, IR-PTV, and LR-PTV were almost equally good for all IMRT and VMAT with and without JT plans. The quality of the plan remains almost same for the all the PTV's.

Jaw tracking methods have also been used in treatment modalities aiming to achieve better target coverage and critical structure sparing. Feng et al. [5] found both JT-IMRT and SJIMRT plans can achieve comparable target dose coverage. Our results are shows both JT and SJ IMRT/VMAT method has a comparable target conformity and homogeneity with appreciable OAR sparing. JT displays superior OARs sparing than SJ plans and they are of clinical importance, especially for the patients with large and complex targets but close to some highly radio-sensitive organs to spare. The mean low doses reduction for OARs ranged from $0.5 \%$ to $7.64 \%$ in our study. The JT-IMRT plans deposited significantly mean lower dose to various OARs, the mean doses reductions for these OARs and the value reductions depend on the volume and the location of the OARs. In JT-IMRT periphery OAR has an lesser low dose and mean dose reduction compared to the OAR in the center or close to the PTV's, the maximum of $7.64 \%$ reduction in the periphery structure of parotid compare to $1.4 \%$ reduction in the thyroid.

Snyder et al. [7] evaluated dosimetric benefits of JT for spine stereotactic radiosurgery using IMRT and VMAT. It showed that, Jaw tracking decreased spinal cord for both IMRT and VMAT plans, but a larger decrease 10\% was seen with the IMRT plans ( $p=0.004$ vs. $p=0.040$ ).

Joy et al. [8] evaluated the dosimetric effects of JT in stepand-shoot IMRT, showing an overall reduction of normal tissue doses. Most patients had reductions of $V_{51} V_{10}$, and $V_{20}$ by less than $2 \%$ in the normal tissues. The maximum reduction in $V_{5}$ was $16.7 \%$. In our study we found for the volume of $V_{5}, V_{10}$ and $V_{20}$ an average reduction of $4.69 \%, 2.44 \%$, and $2.12 \%$ for JT-IMRT compare to SJ-IMRT, whereas a reduction of $2.75 \%$, 2.06\%, and 1.06\% were found between JT-VMAT and SJ-VMAT.

Kim et al. [9] assessed the potential advantages of JT technique by using control point sequences of VMAT planning, showing that, for HetN cases, the mean dose reductions for all the OARs ranged from $4.3 \%$ to $11.9 \%$. In prostate patients, the organs far from the target showed larger sparing (3.7\% to 8.1\% on average) in JT than the organs adjacent to the target (1.1\% to $1.5 \%$ ). The dose reductions were more significant in the dose regions of $D_{80}, D_{90}$ and $D_{95}$ for all the patients in JT plans.

Schmidhalter et al. [10] evaluated the leaf transmission reduction using moving jaws in dynamic MLC IMRT, and demonstrated that the undesired doses to the body volume minus the PTV decreased by up to $1.8 \%$ and $1.5 \%$ for prostate and HEN patients. Simultaneously, the monitor unit (MU) increased by up to $3.1 \%$ and $2.8 \%$, respectively. In our case the low dose volume $V_{5}$ reduced to $4.69 \%$ and $2.75 \%$ for the JT-IMRT and JT-VMAT compared to the SJ-IMRT and SJ-VMAT. The total MU $\left(\bar{x} \pm \sigma_{\bar{x}}\right)$ for all the patients for JT-IMRT, SJ-IMRT, JT-VMAT, and SJ-VMAT were 1,385 $\pm 266,1,329 \pm 226,484$ \pm 58 , and $486 \pm 58$. The MU increased in JT-IMRT by $4.21 \%$ compared to SJ-IMRT, but there is no difference in the total MU for the JT-VMAT and SJ-VMAT plans. The design of the Varian $M L C$ requires fields $>14 \mathrm{~cm}$ in width to be split into 2 or more carriage movements [11]. With the split-field technique, both the number of MUs and total treatment time are significantly increased [11]. Since we used smart leaf motion calculator (LMC) ver. 10.0, there is no split of larger field sizes. If the same study simulated with the older LMC instead of the smart LMC, both the MU and treatment time is going to increases significantly and end up in increased lower dose volumes and OAR doses in the SJ-IMRT technique.

Head and neck site itself large and complex target surrounded by many critical tissues and sensitive structures such as spinal cord, brainstem, thyroid, inferior constrictor, and parotid glands. This study demonstrates that the mean values of the maximum and mean doses to the spinal cord, brainstem, and parotid are significantly reduced in the IMRT and VMAT with JT plan in comparison of without JT plan as shown in Tables 4 and 5. Perhaps these OARs do not overlap with the target in the selected cases, yet the thyroid and inferior constrictor are partially or entirely covered by PTVs so dose reduction in these OARs is slightly lower. The dose reduction to the OARs (parotid glands, spinal cord, etc.), and normal tissue may reduce risk of radiation induced injury and complication such as xerostomia $[12,13]$. JT technique is also more meaningful to reduce the low dose volume for the radiotherapy patients with local recurrent or second primary malignant lesion within or adjacent to a previously irradiated area.

The risk of radiation induced secondary malignancies is another concern, which is strongly associated with low dose exposure of normal tissues during IMRT/VMAT. The JT technique significantly reduces the low dose regions. In the HEtN cases, the JT (IMRT/NMAT) technique significantly reduces the $V_{5}, V_{10}, V_{20}, V_{30}$, and mean dose to the normal tissue body, compared to the SJ (IMRT/VMAT). The maximum reduction was observed in $V_{5}$ and minimum at $V_{30}$ as shown in Tables 4 and 5.

In our study we also found that the JT-IMRT plan gives 
significantly lower mean doses to all OARs, and also significantly reduction in low dose receiving volume $V_{51}, V_{10}, V_{20}$ and $V_{30}$ which are shown in Table 4. The mean doses of OARs have been widely used to predict the probability of radiation toxicity [12] like xerostomia. Therefore, JT plans may decrease the probability of developing acute and late side effects or secondary neoplasm.

In overall analysis of our study shows that, JT-IMRT plans gives superior dose reduction in all OARs and in the low dose volume then that of JT-VMAT plans, may be this is due to high monitor unit used in IMRT in comparison to VMAT plans.

In conclusion, this study demonstrates that all the JTIMRT and SJ-IMRT plans can achieve comparable target dose coverage and are clinically acceptable. JT-IMRT displays superior OARs sparing compare to (static) IMRT plans. These results are of clinical importance, especially for the patients with large and complex targets, but close to some highly radio-sensitive organs to spare, and for patients with local recurrent or secondary primary malignant lesion within a previously irradiated area. Also the JT-IMRT plan significantly reduces the low dose volume compare to the without JT-IMRT plan. JT plans may decrease the probability of developing late side effects or secondary neoplasm.

In comparison of overall plan, JT-VMAT shows insignificant results compare to SJ-VMAT plans in OARs and low dose volumes sparing. The JT-VMAT plans were not clinical significant might be due to less amount of MU compare to IMRT and the delivery method of JT-VMAT.

\section{Conflict of Interest}

No potential conflict of interest relevant to this article was reported.

\section{References}

1. Lee N, Puri DR, Blanco Al, Chao KS. Intensity-modulated radiation therapy in head and neck cancers: an update. Head Neck 2007;29:387-400.

2. Ng J, Shuryak I. Minimizing second cancer risk following radiotherapy: current perspectives. Cancer Manag Res 2014;7:1-11.

3. LoSasso T, Chui CS, Ling CC. Physical and dosimetric aspects of a multileaf collimation system used in the dynamic mode for implementing intensity modulated radiotherapy. Med Phys 1998;25:1919-27.

4. Cadman P, McNutt T, Bzdusek K. Validation of physics improvements for IMRT with a commercial treatmentplanning system. J Appl Clin Med Phys 2005;6:74-86.

5. Feng Z, Wu H, Zhang $Y$, Zhang $Y$, Cheng J, Su X. Dosimetric comparison between jaw tracking and static jaw techniques in intensity-modulated radiotherapy. Radiat Oncol 2015;10:28.

6. International Commission on Radiation Units and Measurements. Prescribing, recording, and reporting photonbeam intensity-modulated radiation therapy (IMRT): contents (ICRU Report 83). J ICRU 2010;10:NP.

7. Snyder KC, Wen N, Huang $Y$, et al. Use of jaw tracking in intensity modulated and volumetric modulated arc radiation therapy for spine stereotactic radiosurgery. Pract Radiat Oncol 2015;5:e155-62.

8. Joy S, Starkschall G, Kry S, et al. Dosimetric effects of jaw tracking in step-and-shoot intensity-modulated radiation therapy. J Appl Clin Med Phys 2012;13:136-45.

9. Kim Jl, Park JM, Park SY, Choi CH, Wu HG, Ye SJ. Assessment of potential jaw-tracking advantage using control point sequences of VMAT planning. J Appl Clin Med Phys 2014;15:160-8.

10. Schmidhalter D, Fix MK, Niederer P, Mini R, Manser P. Leaf transmission reduction using moving jaws for dynamic MLC IMRT. Med Phys 2007;34:3674-87.

11. Lee CC, Wu A, Garg M, et al. A new approach to reduce number of split fields in large field IMRT. Med Dosim 2011;36:1-5.

12. Chao KS, Deasy JO, Markman J, et al. A prospective study of salivary function sparing in patients with head-and-neck cancers receiving intensity-modulated or three-dimensional radiation therapy: initial results. Int J Radiat Oncol Biol Phys 2001;49:907-16.

13. Trotti A. Toxicity in head and neck cancer: a review of trends and issues. Int J Radiat Oncol Biol Phys 2000;47:1-12. 